\title{
MicroRNAs: Biogenesis, Roles for Carcinogenesis and as Potential Biomarkers for Cancer Diagnosis and Prognosis
}

\author{
Nowroji Kavitha ${ }^{1}$,Soundararajan Vijayarathna ${ }^{1}$,Subramanion Lachumy Jothy ${ }^{1}$, \\ Chern Ein Oon ${ }^{1}$, Yeng Chen ${ }^{2}$, Jagat Rakesh Kanwar ${ }^{3}$, Sreenivasan Sasidharan ${ }^{1 *}$
}

\begin{abstract}
MicroRNAs (miRNAs) are short non-coding RNAs of 20-24 nucleotides that play important roles in carcinogenesis. Accordingly, miRNAs control numerous cancer-relevant biological events such as cell proliferation, cell cycle control, metabolism and apoptosis. In this review, we summarize the current knowledge and concepts concerning the biogenesis of miRNAs, miRNA roles in cancer and their potential as biomarkers for cancer diagnosis and prognosis including the regulation of key cancer-related pathways, such as cell cycle control and miRNA dysregulation. Moreover, microRNA molecules are already receiving the attention of world researchers as therapeutic targets and agents. Therefore, in-depth knowledge of microRNAs has the potential not only to identify their roles in cancer, but also to exploit them as potential biomarkers for cancer diagnosis and identify therapeutic targets for new drug discovery.
\end{abstract}

Keywords: Cell cycle control - cancer - miRNA dysregulation - oncogene

Asian Pac J Cancer Prev, 15 (18), 7489-7497

\section{Introduction}

MicroRNAs (miRNAs) are small, single stranded and genomically encoded RNA molecules of approximately 19 to 25 nucleotides in length that are found in all higher eukaryotes including mammals, fungi and plants. miRNA lin-4 was the first miRNA discovered in 1993, which regulates the developmental timing of larva by translation repression of the gene lin-14 in the nematode Caenorhabditis elegans (Lee et al., 1993). The discovery of miRNA was proven to be continued when a second miRNA, let-7, which was discovered in 2000 (Reinhart et al., 2000), repressed lin-41, lin-14, lin-28, lin-42, and daf-12 expression during alteration of the developing stage in C. elegans. Subsequently, let-7 was found to be homologous in other species, including humans, and revealed that the existence of miRNAs is quite common in eukaryotes, which is the one of the most exciting scientific breakthroughs in the last decade. Since then, thousands of non-coding RNAs have been studied and 940 distinct miRNA molecules that exist in human genomes were identified and catalogued (Berezikov et al., 2005; Griffiths-Jones et al., 2008; Baumhoer et al., 2012; Chaudhry and Lhakhang, 2012). Each miRNA is predicted to have hundreds of mRNA targets caused by the imperfect base pairing (Lim et al., 2005) to the
3' untranslated region (UTR) of the target mRNAs and signalling the target for mRNA degradation. Thus, miRNAs have been identified as performing significant regulatory functions in various cellular, biological and pathological processes, including the differentiation, progression, apoptosis, and proliferation of cancer cells (Heneghan et al., 2010; Farooqi et al., 2014). These molecules characteristically moderate the translation and stability of mRNAs, including those genes that mediate processes in carcinogenesis, including the immune response, metabolism, inflammation, cell cycle control, viral replication, stem cell differentiation and human development (Farazi et al., 2013).

miRNAs, known as gene regulators, expressed more than $30 \%$ of protein-coding genes in the human genome at the post transcriptional stage and simultaneously targeted multiple genes in the initiation and progression of human malignancies (Croce, 2009). Recent studies also indicated the differences of miRNA expression profiling between tumour and normal cells that revealed that miRNAs are involved in the pathogenesis of all types of human cancer (Heneghan et al., 2010; Kobayashi et al., 2012; Su et al., 2013). Furthermore, miRNAs play numerous roles as tumour suppressors due to the aberrant expression, which can lead to carcinogenesis by inhibiting the malignant potential, or react as an oncogene by triggering malignant

${ }^{I}$ Institute for Research in Molecular Medicine (INFORMM), Universiti Sains Malaysia, Penang, Malaysia, ${ }^{2}$ Dental Research \& Training Unit, and Oral Cancer Research and Coordinating Centre (OCRCC), Faculty of Dentistry, University of Malaya, Kuala Lumpur, Malaysia, ${ }^{3}$ Nanomedicine-Laboratory of Immunology and Molecular Biomedical Research (NLIMBR), School of Medicine (SoM), Molecular and Medical Research (MMR) Strategic Research Centre, Faculty of Health, Deakin University, Geelong, Pigdons Road, Waurn Ponds, Geelong, Victoria, Australia*For correspondence: srisasidharan@yahoo.com 
Nowroji Kavitha et al

potential, or combined interaction in certain cases (NanaSinkam and Croce, 2011; Kong et al., 2012). The various expressions of miRNA genes in cancer tissues may contribute to several factors including affecting the location of miRNA during the mapping of cancer-associated genomic regions, imperfect function of the miRNA, and epigenetic mechanisms that contribute to multifarious miRNA-mediated gene network dysregulation. In contrast, the unique expression and biological properties of different miRNAs in various cancer types can act as novel bio-markers from which a new strategy might be developed for microRNA gene therapy as a diagnostic and prognostic tool in cancer research (Stenvang et al., 2008; Iorio and Croce, 2012a; Iorio et al., 2012; Corsini et al., 2012; Fink and Kishore Guda, 2013). In this review, we have reported the biogenesis of miRNAs, miRNAs roles in cancer and its potential as biomarkers for cancer diagnosis and prognosis including the regulation of key cancer-related pathways, such as cell cycle control and the miRNA dysregulation. Such depth information has the potential not only to identify the roles of microRNAs in cancer, but also to exploit microRNAs as potential biomarkers for cancer diagnosis and identify therapeutic target for new drug discovery.

\section{miRNA Biogenesis}

More recently, many researchers have determined that microRNAs play an integral role as a sophisticated modulator and have demonstrated the evolutionary importance in the miRNA biogenesis process, function and degradation by a range of mechanisms involving numerous protein-protein and protein-RNA interactions (Kim et al., 2009; Krol et al., 2010). Hence, understanding the miRNA biogenesis pathway is crucial due to the multiple links between human diseases, especially cancers, and the irregular expression of miRNAs or deficiency in miRNA biogenesis (Iorio and Croce, 2012a). Most miRNA genes are located in intergenic regions, which have been predicted from annotating regions in the genome data software, and can be found between the exonic and intronic regions in either sense or antisense alignments (Ruby et al., 2007).

In a canonical pathway, miRNA regulation begins with RNA polymerase II-dependent transcription of miRNA molecules in a nucleus by generating long primary miRNA transcripts (pri-miRNA) that fold into a hairpin structure (Carthew and Sontheimer, 2009; Kim et al., 2009). PrimiRNAs with 5'7 methyl-guanosine (m7G) capped and 3 'polyadenylated are cleaved by the Microprocessor Complex, which is attained through catalytic cleavage of the double-stranded stem by the RNase III endonuclease Drosha (van Kouwenhove et al., 2011), while the hairpin is correctly positioned by co-factor DGCR8 in humans (Di George syndrome Critical Region 8, known as Pasha in D. melanogaster and C. elegans) (Melo and Esteller, 2011a; Melo and Esteller, 2011b). They regulate each other through the response mechanism while DGCR8/ Pasha stabilizes Drosha by interacting with its C-terminal domain and endonuclease cleaves two hairpin structures in the 5'UTR and coding region of Dgcr8 mRNA,

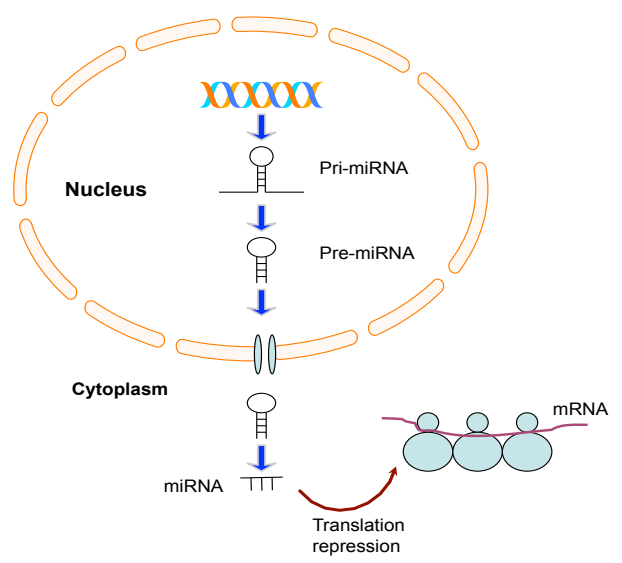

Figure 1. miRNA Biogenesis Pathway

consequently resulting in its degradation (Han et al., 2009; Martinez and Gregory, 2013).

The product from Drosha cleavage is approximately 70 nucleotide (nt) stretches of stem-loop-forming precursor miRNA transcripts (pre-miRNA). Pre-miRNAs, which form short RNA hairpins bearing a 2-nt 3'overhang, are bounded with the nuclear export factor exportin-5 (XPO5 complex protein in with Ran protein) and then shuttled to the cytoplasm along with GTP hydrolysis, known as nucleocytoplasmic transport pathways (Melo and Esteller, 2011b). Once in the cytoplasm, (dsRNA)-specific RNase III endonuclease enzyme, termed Dicer1, together with its catalytic partner human immunodeficiency virus transactivating response RNA-binding protein (TRBP), removes the terminal loop of the pre-miRNA to further process it to an $20-22 \mathrm{nt}$ mature miRNA duplex (Inui et al., 2010).

The miRNA duplex now consists of two 5'phosphorylated sequence strands with 3 'overhangs named miRNA (mature miRNA guide strand) and miRNA* (complementary passenger strand), and recently named as (miR-3p/*miR-5p) (Iorio and Croce, 2012b). The mature miRNAs guide strand, which is generally selected according to its thermodynamic properties, is then loaded into a multi-complex; the RNA-induced silencing complex (RISC) comprising argonaute proteins (AGO2) in which the miRNAs function as post-transcriptional regulators of gene expression through direct base pairing with the target mRNAs (miRISC) (Garzon et al., 2010). The miRNAs specific binding to complementary sequences of mRNAs may either RISC induces mRNA degradation (perfect pairings) or the translation into protein is blocked (imperfect miRNA-mRNA target pairing) was defined by Watson-Crick base pairing between positions 2 to 8 from the 5'miRNA (also known as the seed) with the 3'untranslated region (UTR) of their target mRNAs (Ghildiyal and Zamore, 2009; Bartel, 2009). The formed miRNA with RISC are then transported back into the nucleus to exert its biological effect since a single miRNA is capable of targeting hundreds of mRNAs, which highlights the impact of gene regulation system in cellular functions (Taby and Issa, 2010). The complementary passenger strand (miRNA*), which was initially thought to be degraded and known as a nonfunctional bioproduct of miRNA biogenesis (Gregory et al., 2005), has recently become an interesting revelation 
for researchers inasmuch as new findings suggest that it can be specified as a functional strand. Reviews of the evidence that miRNA* plays a significant role in cellular function have reported on miRNA* activity in human diseases, especially cancer (Bhayani et al., 2012; Iorio and Croce, 2012b).

In a contradictory way, a few pre-miRNAs are processed directly from short introns (mirtrons) bypassing the Drosha-DGCR8 step in the biogenesis pathway. Through the dicer-independent mechanism, the miRNA was cleaved by AGO2 to form a mature miRNA, while some of the miRNAs bind to the 5'-UTR of the target mRNA and lead to translational activation. Then, the complementarity between miRNA and the mRNA target is facilitated by miRISC directed cleavage of the mRNA target but the low complementarity of miRNA-mediated regulation is carried out by translational repression. Therefore, pre- and/or post initiation of translation may occur by leading to gene silencing. The target mRNAs can also be stored in processing bodies or cytoplasmic and the reverse mechanism may occur by re-entry into the polysomes for translation. Finally, the RISC-independent decoy activity, has recently reported that miRNAs can bind to ribonucleoproteins in a seed sequence and a RISC-independent manner and then interfere with their RNA binding functions (Beitzinger and Meister, 2010; Parasramka et al., 2012). Furthermore, the suppression of the key regulators in miRNA biogenesis machinery, such as Drosha, DGCR8, DICER1, TRBP and XPO5, may be dysfunctional or dysregulated in cancer and may promote cellular transformation by enhancing tumourigenesis (Melo et al., 2010; Melo and Esteller, 2011b). Therefore, different vigorous activity and the role of noncoding RNAs in the biogenesis process is of significant importance, especially in cancer biology studies, which may contribute to the development and stimulate various cancerous cells.

\section{MicroRNAs as Oncogenes and Tumour Suppressors}

MicroRNAs can react as a 'tumour suppressor gene', which is a gene that protects a cell from one step on the path to cancer; for example, when lost in tumour, or usually downregulates the expression of an 'oncogene', which is a gene that has the potential to cause cancer. Moreover, in some cases same miRNA (such as miR-205) will acts either as an oncogene via facilitating tumor initiation and proliferation, or as a tumor suppressor through inhibiting proliferation and invasion in different types of cancers (Orang et al., 2014). The loss of functional miRNAs in the biogenesis pathway by mutations, deletions, promoter methylation, inactivation or any abnormalities may lead to an irregular expression of the target oncogene, which can form tumorous cells (Wong et al., 2011). More loss of miRNA expression has been found in various cancerous cells compared to normal cells; the involvement of miRNAs in human cancer, known as oncogenic miRNAs/'oncomir', was first proven in 2002 by Dr Croce's group; miR-15a-miR-16-1 expression was frequently deleted or downregulated in patients with chronic lymphocytic leukaemia (CLL) at
MiRNA Roles in Cancer Development, Diagnosis and Prognosis

chromosome 13q14 (Calin et al., 2008). Subsequently, other findings, such as the loss of the mir-29b-1-mir-29a (Garzon et al., 2009) in leukaemia patients resulted in the upregulation of target oncoproteins, such as B-cell lymphoma protein-2 (BCL-2), myeloid cell leukaemia sequence 1 (BCL-2-related) (MCL1), TCL1, CDK6 and DNA methyltransferase $3 \alpha$ (DNMT3a) (Garzon et al., 2010). In addition, the overexpression of miRNAs, which downregulates the tumour suppressor genes, may also induce cell tumour proliferation, angiogenesis, and invasion by preventing apoptosis and increasing genetic instability. For example, the amplification expression of mir-17, mir-21 and mir-155 relatively cause tumour initiation and progression by inhibiting the expression of tumour suppressor genes, such as phosphatase and tensin homologue (PTEN), and programmed cell death 4 (PDCD4) (Meng et al., 2007; Petrocca et al., 2008; Frankel et al., 2008). In contrast, p53, also known as tumour protein 53 , and is a tumour protein that in humans is encoded by the TP53 gene (McBride et al., 1986), is the most important and well-studied cancer gene that regulates the cell cycle involved in preventing cancer. Fundamentally, the action of p53 is to induce the expression of miRNAs, best described by the miR34 family, which represses genes that can promote proliferation and apoptosis (He et al., 2007). Additional discoveries indicate that p53-regulated miRNAs, such as miR-192, miR-194, miR-215 and miR-605, can target MDM2, which is a negative regulator of p53 (Deng and Sui, 2013). Furthermore, miR-107 and miR-200 inhibit angiogenesis and epithelial-to-mesenchymal transition (Chang et al., 2011), and other miRNAs - miR-15/16 and miR-192/194/215 families, miR-145 and miR-107 - are directly induced by $\mathrm{p} 53$, and provides a pathway for general insights of the miRNA-mediated control of gene expression and the potential therapeutic opportunities for targeting the p53 network (Lujambio and Lowe, 2012; Krell et al., 2013). Besides that, Suzuki and colleagues (2009) contended that p53 can affect miRNA biogenesis by regulating the process of precursor miRNAs when binding to large DROSHA complex. Supporting findings showed that the mutant p53 binds to inactivate p63, which may reduce the expression of DICER 1 and cancer relevant miRNAs (Su et al., 2010). Consequently, the p53 network is a typical example that miRNAs are related to cancerrelevant pathways at multiple stages and are unpredictable. Thus, a better perception of miRNA biology will help to decipher the role and function of other important cancer genes.

\section{miRNAs in Cell cycle Regulation}

The majority of the tumour-suppressor or oncogenic miRNAs associated with targeting the cell cycle regulators have been described as a tumour-related and oncogenic function of the miRNA alteration in cancerous cells in most of the related miRNAs studies. Furthermore, Wan et al. (2012) recently reported that most of the up-regulated genes were related to cell cycling. The cell cycle dissection is regulated by numerous molecular pathways and specific access points (Figure 2). Generally in the process of 
DNA synthesis, transcription of genes are requisite for DNA duplication throughout the period of $\mathrm{S}$ phase and chromosome division during the period of $M$ phase known as mitosis (Malumbres and Barbacid, 2001). In the most of mitogenic pathways, transcriptional induction of D-type cyclins and activation of cyclin-dependent kinases (CDKs) such as CDK4/CDK6 used to be occurred and resulted in an inactivation of retinoblastoma protein (pRB), leading to the inhibition of E2F transcription of cell cycle targeted genes (Bueno and Malumbres, 2011). The first oncogenic miRNA gene, mir-17-92, and miR106b-25 clusters up regulation, lead to the activation of Cyclin-CDK1 complexes regulators (control of cell cycle) and cell cycle progression by targeting p21Waf/Cip1 (cyclin-dependent kinase inhibitor 1 or CDK-interacting protein 1) and inhibit the G1/S phase switch (Fornari et al., 2008). Similarly, up regulation of mir-17-92 and miR21 by targeting PTEN (phosphatase and tensin homolog protein act as a tumour suppressor gene in the regulation of the cell cycle) and inhibit expression of the transcription factor E2F1, whereby miR-221/222 up regulation are targeting p27Kip1 (a cell-cycle regulatory protein that interacts with cyclin-CDK2/-CDK4) and inhibiting cell cycle progression at $\mathrm{G} 1$, promote cellular proliferation and are capable of up regulating the components of the apoptotic signalling pathway and transforming to cancer cells resistant or susceptible to extrinsic factors at the same checkpoint (Petrocca et al., 2008). Conversely, oncogenic miRNA; miR-15a, miR-16-1 cluster and related miR-15b, miR-16-2 cluster in CLL patients regulated by E2F1 (a critical downstream target of the pRB) often inactivated in human tumours resulting in dysregulation of E2F activity which have been reported that reduces the levels of cyclin D1, cyclin D3, cyclin E1, and CDK6 was triggers an accumulation of multiple cell cycle-promoting genes and was predicted that both miRNAs contribute to apoptosis by decreasing the levels of the apoptotic inhibitor Bcl-2 at G1/S phase (Ofir et al., 2011). Recent findings determine that knockdown of microRNA-34a decreased the rate of apoptosis caused by PRIMA-1 which is a small molecule that restores tumour suppressor function to mutant p53 to induced apoptosis in the cell

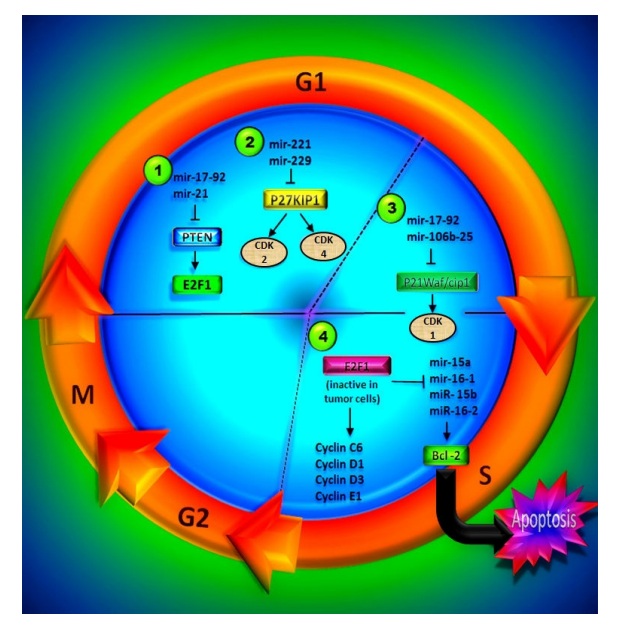

Figure 2. The MiRNA in Cell Cycle Regulation by Numerous Molecular Pathways and Specific Access Points cycle (Duan et al., 2010). Therefore, it can be suggested that microRNA-34a is one of the important components of PRIMA-1-induced apoptotic network in the cancer cells harbouring mutant p53. In addition, the latest findings focused on differentially expressed miRNAs (DEmiRs) involvement in the cell cycle where the specific functions of the p53 pathway might be affected by DEmiRs in cell cycle arrest and apoptosis. They summarised that a group of 26 DEmiRs were found (23 up-regulated and 3 down-regulated) including hsa-miR-21-5p, hsa-miR148a-5p, hsa-miR-181a-5p, hsa-miR-323-3p, and hsamiR-487b-3p, as greatly expressed, and hsa-miR-19a-5p and hsa-miR-25-5p, as down-regulated DEmiRs and the computational analysis revealed that these DEmiRs might only regulate four annotated pathways which are cell proliferation, cell cycle, p53 and TGF-beta/BMP. Moreover, they concluded that, hsa-miR-21-5p, hsamiR-135b-5p and hsa-miR-181c-5p might be regulating cell proliferation and affecting the PTEN by negatively regulating $\mathrm{Akt} / \mathrm{PKB}$ and Notch and/or Wnt signaling pathway in cell cycle which can be associated with carcinogenesis (Sanchez-Diaz et al., 2013). Apart from that, miR-369-3 or let-7, related family members activate the translation in inactive cell lines but suppress the translation in proliferating cells (Vasudevan et al., 2007) which indicates that miRNAs have the possibility in up regulation or down regulation of the translation depending on the cell cycle stage of the cells. All the findings about miRNAs in cell cycle regulation, including modulation in cell-cycle entry and progression, control of miRNAs in cell cycle of tissue homeostasis, and proliferative diseases, have been given emphasis for intense research in the upcoming years in order to understand the connections between miRNAs and the cell division cycle.

\section{miRNA Dysregulation}

miRNA dysregulation is caused by several mechanisms in human cancer including DNA copy number amplification or deletion (Calin and Croce, 2006), genetic mutations, polymorphisms (SNPs), epigenetic mechanism (Lujambio et al., 2008) and deficiency of the key regulators in the miRNA biogenesis pathway (Iorio and Croce, 2012a).

miRNAs often exist in particular genomic regions including the minimal region of the loss of heterozygosity, which may harbour tumour suppressor genes; a minimal region of amplification, which might consist of oncogenes known as fragile sites prone to modification in cancer. Early in 2004, Calin reported that around 50\% of miRNAs are located at fragile sites and cancer susceptibility loci, which are preferred for mature chromatid exchange, translocation, deletion, amplification of plasmid DNA and insertion of tumour associated viruses (Calin and Croce, 2006; Croce et al., 2010; Croce, 2013). The dysregulation of miRNAs expression can be attributable to react critically in tumourigenesis either by inhibiting tumour suppressor genes or activating oncogenes incongruously, thereby contributing to oncogenesis and cancer progression. Previous findings proved that miRNA expression is mutually related to miRNA copy number and tightly overlap with the miRNA expression; for example, 
miR-15a/16 cluster of CLL, located in instable genomic regions embedded into 13q14, which often harbour 13q14 deletions, showed a decreased expression of miR-15a and miR-16. The deletion of $5 q 33$ region contributed to decrease the levels of miR-143 and miR-145 expression in lung cancer. The alteration in miRNA seed sequence caused by mutation has the potential to remove target repression by tumour-suppressive miRNAs or allowing the modification of target selection, which may lead to oncogenesis. Moreover, normally occurring sequence variations, such as SNPs, also influenced miRNA targeting in cancer-related pathways (Wynendaele et al., 2010). These findings suggested that the location of miRNAs in a genomic region responsible for the alterations of miRNAs in a specific type of cancer might participate in the cause of the malignancy in human.

Other recent findings, which indicated that epigenetic aberrations affect miRNA expression by DNA hypermethylation of the tumour suppressor genes, extensive genomic DNA hypomethylation and the alteration of histone modification patterns, explained the dysregulation of miRNA in cancer (Lopez-Serra and Esteller, 2011). From an extensive analysis of genomic sequences, large proportions of miRNA loci are associated with $\mathrm{CpG}$ islands suggesting that miRNAs can represent candidate targets of the DNA methylation machinery. For example, miR-21 and miR-203, which were implanted in a region with $\mathrm{CpG}$ islands, equally decreased miR-124a expression, which was attributed to DNA hypermethylation in colon, breast, and lung carcinomas (Lujambio et al., 2008). Other studies have experimentally proven that potentially oncogenic miRNAs can be upregulated by DNA hypomethylation (Iorio et al., 2005), such as upregulation of hypermethylated tumour suppressing miRNAs by 5-aza-20deoxycytidine treatment in the case of as miR-127, miR-9-1 and miR-34b/c cluster (Toyota et al., 2008). Histone acetylation also represents a similar mechanism of epigenetic alteration in cancer by reducing the level of acetylated histones, which can diminish the expression of anti-oncogenic miRNAs by utilising histone deacetylase inhibitors; alteration of the miRNA levels have been observed during the treatment, which was among the evidence defined by Saito and colleagues (2006). Furthermore, they elaborated that the inhibition of DNA methylation and histone deacetylation only stimulates the expression of miR-127 in cancer cells and that the miR-127 and miR-124a genes affects the expression of two oncogenic proteins BCL6 and CDK6. Subsequently, restoration of miR-29 expression in lung cancer cells downregulates the repression of tumour suppressor genes silenced by $\mathrm{CpG}$ island methylation (Fabbri et al., 2007) and miR-101 targets the histone methyltransferase $\mathrm{EZH} 2$, which focuses more on epigenetic silencing of the target genes and regulates the survival and metastasis of cancer cells (Varambally et al., 2008). The latest findings reviewed by Iliopoulos et al. (2010) indicated that SUZ12 is a component of the polycomb repressor complex 2(PRC2) with EZH2 being the catalytic subunit targeted by the miR-200 family, which seems to be significantly important in the formation of cancer stem cells where the loss of miR-200
MiRNA Roles in Cancer Development, Diagnosis and Prognosis

increases SUZ12 expression, SUZ12 binding, H3-K27 trimethylation, and Polycomb-mediated repression of the E-cadherin gene. The epigenetic changes conjoined by genetic inactivation due to mutation or deletion might be a potential mechanism that is partially involved in miRNA dysregulation of cancer cells.

The inhibition of miRNA biogenesis by suppression of miRNAs processing machinery key regulators including Drosha, DGCR8, DICER1, TRBP and XPO5 leads to severe developmental defects, and promotes cellular transformation and tumourigenesis (Kumar et al., 2009; Melo et al., 2010). However, the mechanism remains unclear and ongoing research in this particular area suggested that miRNAs might have an intrinsic function in tumour suppression and that its downregulation eventually accelerates oncogenesis. Mutations that inactivate XPO5, known as nuclear export receptors, function in premiRNAs transit from the nucleus into the cytoplasm in human tumours, which leads to precursor accumulation in the nucleus and lower levels of mature miRNAs. The inefficient nuclear export and the presence of an inactivating mutation in the exportin-5 gene prevent the formation of a functional XPO5/RAN/GTP/pre-miRNA complex in the miRNA biogenesis pathway. Melo and friends also reported that frameshift mutations in the XPO5 gene in two MSI+ cell lines and primary tumours that have been found in exon 32 alter and abbreviate the protein sequence and prevent XPO5 from associating with its pre-miRNA cargo and exiting the nucleus (Melo et al., 2010). In XPO5 heterozygous mutant cells, less pre-miRNA was available to process in the cytoplasmic machinery, resulting in degradation of the mature miRNA and promoting tumourigenicity (Melo and Esteller, 2011a). In addition, Kumar and colleagues (2009) stated that $27 \%$ of different tumours consist of hemizygous deletion of the gene that encodes DICER1. Global knockdown of mature miRNA production by the depletion of any of the miRNA processing regulators, such as Drosha, RNase, DCGR8 or Dicer, has been shown to promote oncogenesis (Jansson and Lund, 2012). Apparently DICER1 and TARBP is a haploinsufficient tumour suppressor, requiring partial deletion for its associated tumourigenesis phenotype; for example, the mutations of TARBP that damage the miRNA processor and mutations of XPO5 that trap miRNA transcripts in the nucleus, correlate with carcinomas characterised by microsatellite instability (Melo et al., 2009; Melo et al., 2010). Moreover, some studies showed that TARBP is mutated in colon and gastric cancers, which correlates with DICER1 destabilization in the cell line and xenografts with TARBP mutations, thereby reintroduction of wild-type TARBP2/DICER1 slowed tumour growth (Melo et al., 2009). The tumour suppressor p53 is a key regulator of miRNAs with anti-oncogenic roles capable of modulating miRNA expression by the transcription factor and affecting miRNA biogenesis in a transcriptionindependent manner. For example, p53 has been described as modulating miRNA processing through interaction with the Drosha processing complex (Suzuki et al., 2009), while other members of the miR-103/107 family have been shown to target Dicer by reducing the expression of miRNA levels (Martello et al., 2010) in cancer cells. 
A few of the latest discoveries of dysregulation miRNAs in biogenesis machinery have been published; for example, miRNA bindings with competing endogenous RNAs (ceRNAs) network may furnish the chance of an additional level of post-transcriptional regulation and reassessment of the existing regulatory pathways involved in cancer initiation and progression microRNAs ( $\mathrm{Su}$ et al., 2013). The mechanisms resulted in alteration of the expression of UTRs in transcripts and influence miRNA response elements (MRE) levels. Alteration in the MREs of ceRNAs affect the capability of the mRNA transcript to attach miRNAs where the disturbed ceRNA network may contribute to diseases and cancers (Salmena et al., 2011; Karreth et al., 2011). Subsequently, in another latest finding, Shen et al. (2013) proved that the epidermal growth factor receptor (EGFR) directly interacts with argonaute2 (AGO2), a crucial component of the RNAinduced silencing complex (RISC) and prevents cell transformation of specific tumour suppressive miRNAs under hypoxic conditions. Hypoxic stress induces the endocytic trafficking of EGFR into cytoplasm, where EGFR interacts with AGO2 to reduce the binding of Dicer to AGO2 by phosphorylating AGO2-Y393, which results in weakening the process of pre-miRNAs to mature miRNAs (Nishida et al., 2013). Therefore, the perception of mechanisms behind miRNAs dysregulation in human cancer and noteworthy functions might render new insights for improving the classification, prognosis prediction and in the treatment of cancer.

\section{MicroRNAs as Potential Biomarkers for Cancer Diagnosis and Prognosis}

The accumulated studies and analysis on miRNA expression levels in tumourigenesis have determined that miRNAs play a huge role as prognostic and/or diagnostic markers and promising candidates to distinguish different tumour types as well as for predicting the clinical behaviour. The most significant up-to-date findings of miRNAs in cancer concentrate more on the potential of cancer-related miRNAs (oncomiRs) as biomarkers for diagnosis (Lin et al., 2014), prognosis and therapy. Furthermore, therapeutic approaches involving the re-introduction of miRNAs lost in cancer or inhibition of oncogenic miRNAs are rapidly being developed. Additionally, the antisense technology based on antagomiRs which can inhibit the activity of oncogenic miRNAs, is the major method used to reduce unwanted miRNA expression in tumor cells (Mollaie et al., 2013). Recently Xu et al. (2012) reported that multitarget anti-microRNA antisense oligonucleotide (MTgAMOs) can specifically inhibit the expression of multiple miRNAs, and effectively antagonize proliferation and migration of gastric cancer cells promoted by oncomirs. They also suggested that in future, MTg-AMOs can not only be used as an ideal choice of clinical anti-tumor drugs, but can also serve as an important tool for exploring the functions of miRNAs in a number of human tumor types. Also, miRNAs are becoming promising targets for new drug developments in that advanced research could correct the miRNAs regulation by the loss of function or gain of function of miRNAs, which would influence the initiation and progression of cancer (Zhao et al., 2014). Besides that, regulating the gene network could change the behaviour of the cancer cells.

Several diagnostic tools have been implemented for the detection and quantification of microRNA expression levels, such as northern blotting, semiquantitative in situ hybridization, quantitative real-time PCR, fluorescencelabelled bead technology, and array hybridization with various probe sets and alternative protocols and methods for microRNA profiling of FFPE specimens, which are based on real-time PCR or microarray-hybridization (Lawrie, 2008; Streichert et al., 2011; Khan et al., 2011; Streichert et al., 2012; Josephine Wu et al., 2013). For example, the basic MiRNA profiling that identified that the induction of many miRNAs was coincident with the different stages of differentiation was proved by Volinia and colleagues (Volinia et al., 2006) using hierarchical clustering analysis of 540 samples including 363 from six of the most frequent solid tumour types (breast, colon, lung, pancreas, stomach, and prostate). Furthermore, 177 normal controls showed that a unique miRNA signature enabled the tumour samples to be grouped on the basis of their tissue of origin. Again, recently, they have experimentally proven the mechanism, especially in breast cancer by using deep sequencing of microRNA, which also highlighted the prognostic role of these small RNAs in a large set of samples (Croce et al., 2012; Volinia et al., 2012). Nevertheless, each of the approaches has its own specific advantages and disadvantages. Recently, the findings of miRNAs in body fluids, such as serum, urine and colostrum (Hanke et al., 2010; Link et al., 2010) have strengthened investigations into the use of miRNAs as non-invasive biomarkers of disease and a therapeutic response in a range of cancers including lung cancer, ovarian cancer, colorectal cancer, renal cancer, glioblastoma, prostate cancer and breast cancer (Cortez et al., 2012). In recent times, Lin et al. (2014) characterized miRNAs expression profiles in cervical cancer of Uygur women to identify the biomarkers for early diagnosis. Their data showed that the expression of Cox- 2 protein level was significantly increased in cervical cancer. The levels of miR-101 and Cox-2 were negatively correlated in cervical cancer of Uyghur women, which may serve as biomarkers of diagnosis markers for cervical cancer in Uyghur women which provide direct experimental evidence for the roles of miRNA in diagnosis of cancer. Meanwhile, blood based tests for screening purposes or disease monitoring would be more suitable as they are minimally invasive, relatively have low cost and can be repeated as well (Ramshankar and Krishnamurthy, 2013). Because of this, Ramshankar and Krishnamurthy (2013) reported that the circulating miRNAs present in both serum and plasma as attractive candidates to be used as cancer biomarkers and could serve as non- invasive biomarkers for early detection and diagnosis of lung cancer.

Evidently, several studies have demonstrated that miRNAs can be responsible for drug resistance because of the significance of miRNAs in cancer management by modifying the expression also targeted as a therapeutic strategy. Accordingly, the delivery modality of miRNAs or anti-miRNAs can be used in therapeutics, either 
individually or in combination with other treatments, which may have either synergistic effects or antagonistic effects that could critically affect the success of the experimental approaches. Chemotherapy is the most well-known treatment for most solid and haematological malignancies. However, in some tumourigenesis it still harbours either acquired or inherent mechanisms for resistance due to miRNAs regulation of multiple biological functions and it seems plausible that miRNA expression may guide the drug response. These statements have been reviewed by Zenz et al. (2009) who found that the low expression of miR-34a in CLL is associated with p53 inactivation due to chemotherapy-refractory disease, which impaired the DNA damage response and apoptosis resistance irrespective of TP53 mutation or $17 \mathrm{p}$ deletion. Hence, miR-34a is shown to have a role in chemotherapy resistance and thus may serve as a marker for poor prognosis for CLL. miRNAs, as circulating biomarkers, as well as a non-invasive measurement of miRNA expression, is an emerging field that is of great interest to both investigators and clinicians. The detection of circulating miRNAs has been depicted recently in several malignancies including colorectal, lung, breast, and ovarian cancer (Hu et al., 2010; Liu et al., 2011). In addition, Mitchell and Tewari (2010) identified that circulating miRNA in serum in stable form, may circulate within exosomes and microvesicles with the potential for cell-cell communication; however, the origin for circulating miRNAs remains unclear. However, more studies are still ongoing to determine whether circulating miRNAs may be used as surrogate markers for disease activity or response to therapy.

\section{Conclusions}

In conclusion, understanding and research are required since only one-third of the human genes are predicted to be directly targeted by miRNAs. In addition, there is still a lot to be discovered when it comes to miRNAs and their regulation of the human genome in clarifying complex network interactions between miRNAs, other classes of ncRNAs, and protein-coding genes and their involvement in the development of diseases, especially cancer. Concisely, therapeutics can take advantage of this new knowledge as well as diagnostics and prognostics, and clinical applications exploiting our understanding of miRNA function will be the next great challenge in cancer research.

\section{Acknowledgements}

Nowroji Kavitha was supported by a MyPhD fellowship from the Ministry of Higher Education, Government of Malaysia, Malaysia.

\section{References}

Bartel DP (2009). MicroRNAs: target recognition and regulatory functions. Cell, 136, 215-33.

Baumhoer D, Zillmer S, Unger K, et al (2012). MicroRNA profiling with correlation to gene expression revealed
MiRNA Roles in Cancer Development, Diagnosis and Prognosis the oncogenic miR-17-92 cluster to be up-regulated in osteosarcoma. Cancer Genet, 205, 212-19.

Beitzinger M, Meister G (2010). MicroRNAs: from decay to decoy. Cell, 140, 612-14.

Berezikov E, Guryev V, Van De Belt J, et al (2005). Phylogenetic shadowing and computational identification of human microRNA genes. Cell, 120, 21-4.

Bhayani MK, Calin GA, Lai SY (2012). Functional relevance of miRNA* sequences in human disease. Mutat Res-Fund Mol M, 731, 14-9.

Bueno MJ, Malumbres M (2011). MicroRNAs and the cell cycle. Biochim Biophys Acta, 1812, 592-601.

Calin GA, Cimmino A, Fabbri M, et al (2008). MiR-15a and miR-16-1 cluster functions in human leukemia. Proc Natl Acad Sci USA, 105, 5166-71.

Calin GA, Croce CM (2006). MicroRNA signatures in human cancers. Nat Rev Cancer, 6, 857-66.

Carthew RW, Sontheimer EJ (2009). Origins and mechanisms of miRNAs and siRNAs. Cell, 136, 642-55.

Chang CJ, Chao CH, Xia W, et al (2011). p53 regulates epithelialmesenchymal transition and stem cell properties through modulating miRNAs. Nat Cell Biol, 13, 317-23.

Corsini LR, Bronte G, Terrasi M, et al (2012). The role of microRNAs in cancer: diagnostic and prognostic biomarkers and targets of therapies. Expert Opin Ther Tar, 16, 103-9.

Cortez MA, Welsh JW, Calin GA (2012). Circulating microRNAs as noninvasive biomarkers in breast cancer. Rec Res Cancer Res, 195, 151-61.

Croce CM (2013). Ultraconserved regions encoding ncRNAs. US Patent App. 2013, 13/910,410.

Croce CM, Liu CG, Calin GA, Sevignani C (2010). Diagnosis and treatment of cancers with microRNA located in or near cancer associated chromosomal features. Patents, US20100234241 A1.

Croce CM: Causes and consequences of microRNA dysregulation in cancer (2009). Nat Rev Genetics, 10, 704-14.

Deng G, Sui G (2013). Noncoding RNA in oncogenesis: a new era of identifying key players. Int J Mol Sci, 14, 18319-49.

Duan W, Gao L, Wu X, et al (2010). MicroRNA-34a is an important component of PRIMA-1-induced apoptotic network in human lung cancer cells. Int J Cancer, 127, 313-20.

Fabbri M, Garzon R, Cimmino A, et al (2007). MicroRNA-29 family reverts aberrant methylation in lung cancer by targeting DNA methyltransferases 3A and 3B. Proc Natl Acad Sci USA, 104, 15805-10.

Farazi TA, Hoell JI, Morozov P, Tuschl T (2013). MicroRNAs in human cancer. Adv Exp Med Biol, 774, 1-20.

Farooqi AA, Qureshi MZ, Coskunpinar E, et al (2014). miR-421, miR-155 and miR-650: Emerging trends of regulation of cancer and apoptosis. Asian Pac J Cancer Prev, 15, 1909-12.

Fink SP, Kishore Guda D(2013) Application of next-generation sequencing in RNA biomarker discovery in cancer research, In next generation sequencing in cancer research, Wu, Wei, Choudhry, Hani (Eds.), Springer, 1, 383.

Fornari F, Gramantieri L, Ferracin M, et al (2008). MiR-221 controls CDKN1C/p57 and CDKN1B/p27 expression in human hepatocellular carcinoma. Oncogene, 27, 5651-61.

Frankel LB, Christoffersen NR, Jacobsen A, et al (2008). Programmed cell death 4 (PDCD4) is an important functional target of the microRNA miR-21 in breast cancer cells. J Bio Chem, 283, 1026-33.

Garzon R, Heaphy CE, Havelange V, et al (2009). MicroRNA $29 \mathrm{~b}$ functions in acute myeloid leukemia. Blood, 114, 5331-41.

Garzon R, Marcucci G, Croce CM (2010). Targeting microRNAs in cancer: rationale, strategies and challenges. Nat Rev Drug 
Discovery, 9, 775-89.

Ghildiyal M, Zamore PD (2009). Small silencing RNAs: an expanding universe. Nat Rev Genet, 10, 94-108.

Gregory RI, Chendrimada TP, Cooch N, Shiekhattar R (2005). Human RISC couples microRNA biogenesis and posttranscriptional gene silencing. Cell, 123, 631-40.

Griffiths-Jones S, Saini HK, Van Dongen S, Enright AJ (2008). miRBase: tools for microRNA genomics. Nucleic Acids Res, 36, 154-8.

Han J, Pedersen JS, Kwon SC, et al (2009). Posttranscriptional crossregulation between Drosha and DGCR8. Cell, 136, 75-84.

Hanke M, Hoefig K, Merz H, et al (2010). A robust methodology to study urine microRNA as tumor marker: microRNA-126 and microRNA-182 are related to urinary bladder cancer. In: Urologic Oncology: Seminars and Original Investigations, 11, 655-61.

He L, He X, Lowe SW, Hannon GJ (2007). microRNAs join the p53 network-another piece in the tumour-suppression puzzle. Nat Rev Cancer, 7, 819-22.

Heneghan HM, Miller N, Kerin MJ (2010). MiRNAs as biomarkers and therapeutic targets in cancer. Curr Opin Pharmacol, 10, 543-50.

$\mathrm{Hu}$ Z, Chen X, Zhao Y, et al (2010). Serum MicroRNA signatures identified in a genome-wide serum MicroRNA expression profiling predict survival of non-small-cell lung cancer. $J$ Clin Oncol, 28, 1721-6.

Iliopoulos D, Lindahl-Allen M, Polytarchou C, et al (2010). Loss of miR-200 inhibition of Suz12 leads to polycomb-mediated repression required for the formation and maintenance of cancer stem cells. Mol Cell, 39, 761-72.

Inui M, Martello G, Piccolo S (2010). MicroRNA control of signal transduction. Nat Rev Mol Cell Biol, 11, 252-63.

Iorio MV, Casalini P, Piovan C, Braccioli L, Tagliabue E (2012). Current and future developments in cancer therapy research: mirnas as new promising targets or tools. In Biotargets of Cancer in Current Clinical Practice, Bologna, M. (Ed.), Springer. XVI, 563.

Iorio MV, Croce CM (2012). MicroRNA dysregulation in cancer: diagnostics, monitoring and therapeutics. A comprehensive review. EMBO Mol Med, 4, 143-59.

Iorio MV, Croce CM (2012). microRNA involvement in human cancer. Carcinogenesis, 33, 1126-33.

Iorio MV, Ferracin M, Liu CG, et al (2005). MicroRNA gene expression deregulation in human breast cancer. Cancer Res, 65, 7065-70.

Jansson MD, Lund AH (2012). MicroRNA and cancer. Mol Oncol, 6, 590-610.

Josephine WD, Cui M, Shibata R, Cheng L, Zhang DY (2013). Diagnostic methodology and technology. In Molecular Genetic Pathology, Cheng, Liang, Zhang, David Y., Eble, John N. (Eds.), Springer, 2nd ed., XXI, 1136 p.

Karreth FA, Tay Y, Perna D, et al (2011). In vivo identification of tumor-suppressive PTEN ceRNAs in an oncogenic BRAFinduced mouse model of melanoma. Cell, 147, 382-95.

Khan N, Cheng J, Pezacki JP, Berezovski MV (2011). Quantitative analysis of microRNA in blood serum with protein-facilitated affinity capillary electrophoresis. Anal Chem, 83, 6196-01.

Kim VN, Han J, Siomi MC (2009). Biogenesis of small RNAs in animals. Nat Rev Mol Cell Biol, 10, 126-39.

Kobayashi E, Hornicek FJ, Duan Z (2012). MicroRNA involvement in osteosarcoma. Clin Sarcoma Res, 12, 359739.

Kong YW, Ferland-Mccollough D, Jackson TJ, Bushell M (2012). microRNAs in cancer management. The Lancet Oncol, 13, 249-258.
Krell J, Frampton AE, Colombo T, et al (2013). The p53 miRNA interactome and its potential role in the cancer clinic. Epigenomics, 5, 417-28.

Krol J, Loedige I, Filipowicz W (2010). The widespread regulation of microRNA biogenesis, function and decay. Nat Rev Genet, 11, 597-610.

Kumar MS, Pester RE, Chen CY, et al (2009). Dicer1 functions as a haploinsufficient tumor suppressor. Genes Dev, 23, 2700-4.

Lawrie CH (2008). MicroRNA expression in lymphoid malignancies: new hope for diagnosis and therapy? J Cell Mol Med, 12, 1432-44.

Lee RC, Feinbaum, RL, d Ambros V (1993). The C. elegans heterochronic gene lin-4 encodes small RNAs with antisense complementarity to lin-14. Cell, 75, 843-54.

Lhakhang TW, Chaudhry M (2012). Current approaches to micro-RNA analysis and target gene prediction. J App Gene, 53, 149-58.

Lim LP, Lau NC, Garrett-Engele P, et al (2005). Microarray analysis shows that some microRNAs downregulate large numbers of target mRNAs. Nature, 433, 769-73.

Lin C, Huang F, Zhang Y, Tuokan T, Kuerban G. (2014). Roles of MiR-101 and its target gene cox-2 in early diagnosis of cervical cancer in Uygur women. Asian Pac J Cancer Prev, 15, 45-8.

Link A, Balaguer F, Shen Y, et al (2010). MicroRNAs as novel biomarkers for colon cancer screening. Cancer Epidem Biomar Prev, 19, 1766-74.

Liu R, Zhang C, Hu Z, et al (2011). A five-microRNA signature identified from genome-wide serum microRNA expression profiling serves as a fingerprint for gastric cancer diagnosis. Eur J Cancer, 47, 784-91.

Lopez-Serra P, Esteller M (2011). DNA methylation-associated silencing of tumor-suppressor microRNAs in cancer. Oncogene, 31, 1609-22.

Lujambio A, Calin GA, Villanueva A, et al (2008). A microRNA DNA methylation signature for human cancer metastasis. Proc Natl Acad Sci USA, 105, 13556-61.

Lujambio A, Lowe SW (2012). The microcosmos of cancer. Nature, 482, 347-55.

Malumbres M, Barbacid M (2001). Milestones in cell division: to cycle or not to cycle: a critical decision in cancer. Nat Rev Cancer, 1, 222-31.

Martinez NJ, Gregory RI (2013). Argonaute2 expression is post-transcriptionally coupled to microRNA abundance. RNA, 19, 605-12.

Mcbride O, Merry D, Givol D (1986). The gene for human p53 cellular tumor antigen is located on chromosome 17 short arm (17p13). Proc Natl Acad Sci U.S.A, 83, 130-4.

Melo SA, Esteller M (2011). A precursor microRNA in a cancer cell nucleus: get me out of here! Cell Cycle, 10, 922-5.

Melo SA, Esteller M (2011). Dysregulation of microRNAs in cancer: playing with fire. FEBS Letters, 585, 2087-99.

Melo SA, Moutinho C, Ropero S, et al (2010). A genetic defect in exportin-5 traps precursor microRNAs in the nucleus of cancer cells. Cancer cell, 18, 303-15.

Melo SA, Ropero S, Moutinho C, et al (2009). A TARBP2 mutation in human cancer impairs microRNA processing and DICER1 function. Nat Genetics, 41, 365-70.

Meng F, Henson R, Wehbe-Janek H, et al (2007). MicroRNA-21 regulates expression of the PTEN tumor suppressor gene in human hepatocellular cancer. Gastroenterology, 133, 647-58.

Mitchell PS, Tewari M (2010). Circulating microRNAs in cancer. In Kikuchi Y. and Rykova EY. (Eds.), Nucleic acids and molecular biology series: extracellular nucleic acids, Springer, 129-46.

Mollaie HR, Monavari SH, Arabzadeh SA, et al (2013). RNAi 
and miRNA in viral infections and cancers. Asian Pac $J$ Cancer Prev, 14, 7045-56.

Nana-Sinkam SP, Croce CM (2011). MicroRNAs as therapeutic targets in cancer. Transl Res, 157, 216-25.

Nishida N, Mimori K, Mori M, Calin GA (2013). EGFR gets in the way of microRNA biogenesis. Cell Research, 23, 1157-8.

Ofir M, Hacohen D, Ginsberg D (2011). MiR-15 and miR-16 are direct transcriptional targets of E2F1 that limit E2F-induced proliferation by targeting cyclin E. Mol Cancer Res, 9, 440-7.

Orang AV, Safaralizadeh R, Hosseinpour Feizi MA (2014). Insights into the diverse roles of miR-205 in human cancers. Asian Pac J Cancer Prev, 15, 577-583.

Parasramka MA, Ho E, Williams DE, Dashwood RH (2012). MicroRNAs, diet, and cancer: new mechanistic insights on the epigenetic actions of phytochemicals. Mol Carcinogen, 51, 213-30.

Petrocca F, Visone R, Onelli MR, et al (2008). E2F1-regulated microRNAs impair TGF $\beta$-dependent cell-cycle arrest and apoptosis in gastric cancer. Cancer Cell, 13, 272-86.

Ramshankar V, Krishnamurthy A (2013). Lung cancer detection by screening - presenting circulating miRNAs as a promising next generation biomarker breakthrough. Asian Pac J Cancer Prev, 14, 2167-72.

Reinhart BJ, Slack FJ, Basson M, et al (2000). The 21-nucleotide let-7 RNA regulates developmental timing in Caenorhabditis elegans. Nature, 403, 901-6.

Ruby JG, Jan CH, Bartel DP (2007). Intronic microRNA precursors that bypass Drosha processing. Nature, 448, 83-6.

Saito Y, Liang G, Egger G, et al (2006). Specific activation of microRNA-127 with downregulation of the proto-oncogene by chromatin-modifying drugs in human cancer cells. Cancer Cell, 9, 435-43.

Salmena L, Poliseno L, Tay Y, Kats L, Pandolfi PP (2011). A ceRNA hypothesis: the rosetta stone of a hidden RNA language? Cell, 146, 353-8.

Sanchez-Diaz PC, Hsiao TH, Chang JC, et al (2013). Deregulated microRNAs in pediatric cancer stem cells target pathways involved in cell proliferation, cell cycle and development. PloS One, 8, 61622.

Shen J, Xia W, Khotskaya YB, et al (2013). EGFR modulates microRNA maturation in response to hypoxia through phosphorylation of AGO2. Nature, 497, 383-7.

Stenvang J, Silahtaroglu AN, Lindow M, Elmen J, Kauppinen S (2008). The utility of LNA in microRNA-based cancer diagnostics and therapeutics. Semin Cancer Biol, 18, 89-102.

Streichert T, Otto B, Lehmann U (2011). MicroRNA profiling using fluorescence-labeled beads: data acquisition and processing. Methods Mol Biol, 676, 253-68.

Streichert T, Otto B, Lehmann U (2012). microRNA expression profiling in archival tissue specimens: methods and data processing. Mol Biotechnol, 50, 159-69.

Su X, Chakravarti D, Cho MS, et al (2010). TAp63 suppresses metastasis through coordinate regulation of Dicer and miRNAs. Nature, 467, 986-90.

Su X, Xing J, Wang Z, et al (2013). microRNAs and ceRNAs: RNA networks in pathogenesis of cancer. Chin J Cancer Res, 25, 235-9.

Suzuki HI, Yamagata K, Sugimoto K, et al (2009). Modulation of microRNA processing by p53. Nature, 460, 529-33.

Taby R, Issa JPJ (2010). Cancer epigenetics. CA Cancer J Clin, 60, 376-92.

Toyota M, Suzuki H, Sasaki Y, et al (2008). Epigenetic silencing of microRNA-34b/c and B-cell translocation gene 4 is associated with $\mathrm{CpG}$ island methylation in colorectal cancer. Cancer Res, 68, 4123-32.

Van Kouwenhove M, Kedde M, Agami R (2011). MicroRNA regulation by RNA-binding proteins and its implications for cancer. Nat Rev Cancer, 11, 644-56.

Varambally S, Cao Q, Mani RS, et al (2008). Genomic loss of microRNA-101 leads to overexpression of histone methyltransferase EZH2 in cancer. Science, 322, 1695-9.

Vasudevan S, Tong Y, Steitz JA (2007). Switching from repression to activation: microRNAs can up-regulate translation. Science, 318, 1931-4.

Volinia S, Calin GA, Liu CG, et al (2006). A microRNA expression signature of human solid tumors defines cancer gene targets. Proc Natl Acad Sci USA, 103, 2257-61.

Volinia S, Galasso M, Sana ME, et al (2012). Breast cancer signatures for invasiveness and prognosis defined by deep sequencing of microRNA. Proc Natl Acad Sci USA, 109, 3024-9.

Wan SM, Lv F, Guan T (2012). Identification of genes and microRNAs involved in ovarian carcinogenesis. Asian Pac J Cancer Prev, 13, 3997-4000.

Wong KY, Yu L, Chim CS (2011). DNA methylation of tumor suppressor miRNA genes: a lesson from the miR-34 family. Epigenomics, 3, 83-92.

Wynendaele J, Bohnke A, Leucci E, et al (2010). An illegitimate microRNA target site within the 3'UTR of MDM4 affects ovarian cancer progression and chemosensitivity. Cancer Res, 70, 9641-9.

Xu L, Dai WQ, Xu XF, et al (2012). Effects of multiple-target anti-microRNA antisense oligodeoxyribonucleotides on proliferation and migration of gastric cancer cells. Asian Pac J Cancer Prev, 13, 3203-7.

Zenz T, Mohr J, Eldering E, et al (2009). miR-34a as part of the resistance network in chronic lymphocytic leukemia. Blood, 113, 3801-8.

Zhao SF, Zhang X, Zhang XJ, et al (2014). Induction of MicroRNA-9 mediates cytotoxicity of curcumin against SKOV3 ovarian cancer cells. Asian Pac J Cancer Prev, 15, 3363-8. 